\title{
Cochrane
}

Library

Cochrane Database of Systematic Reviews

\section{Occupational therapy delivered by specialists versus non-specialists for people with schizophrenia (Review)}

Morris K, Reid G, Spencer S

Morris K, Reid G, Spencer S.

Occupational therapy delivered by specialists versus non-specialists for people with schizophrenia.

Cochrane Database of Systematic Reviews 2018, Issue 10. Art. No.: CD012398.

DOI: 10.1002/14651858.CD012398.pub2.

www.cochranelibrary.com

Occupational therapy delivered by specialists versus non-specialists for people with schizophrenia (Review) Copyright $\odot 2018$ The Cochrane Collaboration. Published by John Wiley \& Sons, Ltd. 
TABLE OF CONTENTS

HEADER 1

ABSTRACT

PLAIN LANGUAGE SUMMARY

SUMMARY OF FINDINGS

BACKGROUND

OBJECTIVES

METHODS

RESULTS

Figure 1.

DISCUSSION

AUTHORS' CONCLUSIONS

ACKNOWLEDGEMENTS

REFERENCES

CHARACTERISTICS OF STUDIES

ADDITIONAL TABLES

CONTRIBUTIONS OF AUTHORS

DECLARATIONS OF INTEREST

SOURCES OF SUPPORT

DIFFERENCES BETWEEN PROTOCOL AND REVIEW

INDEX TERMS

1

2

3

5

6

6

12

13

14

14

15

16

20

22

24

24

24

24

24 
[Intervention Review]

\section{Occupational therapy delivered by specialists versus non-specialists for people with schizophrenia}

Karen Morris ${ }^{1}$, Graeme Reid ${ }^{2}$, Sally Spencer 3

1Department of Health, Psychology \& Social Studies, University of Cumbria, Carlisle, UK. 2North West Boroughs Healthcare NHS

Foundation Trust, Warrington, UK. ${ }^{3}$ Postgraduate Medical Institute, Edge Hill University, Ormskirk, UK

Contact address: Sally Spencer, Postgraduate Medical Institute, Edge Hill University, St Helens Road, Ormskirk, Lancashire, L39 4QP, UK. spencesa@edgehill.ac.uk.

Editorial group: Cochrane Schizophrenia Group.

Publication status and date: New, published in Issue 10, 2018.

Citation: Morris K, Reid G, Spencer S. Occupational therapy delivered by specialists versus non-specialists for people with schizophrenia. Cochrane Database of Systematic Reviews 2018, Issue 10. Art. No.: CD012398. DOI: 10.1002/14651858.CD012398.pub2.

Copyright @ 2018 The Cochrane Collaboration. Published by John Wiley \& Sons, Ltd.

\section{A B S T R A C T}

\section{Background}

Schizophrenia is a severe mental health condition that is characterised by positive symptoms, such as hallucinations and delusions; negative symptoms, such as flattened affect, thought disorder (disrupted speech), and lack of motivation; and cognitive symptoms, such as problems with memory and attention. Schizophrenia can occur as an isolated episode, or as a recurring cycle of remission and relapse, and is associated with impairment in psychosocial and occupational functioning.

Although antipsychotic drugs are the main treatment for people with schizophrenia, in most countries mental health services usually provide a range of add-on interventions, including occupational therapy. This is a complex intervention designed to support and enable continued participation in daily life through engagement in activities and occupations meaningful to the individual. Occupational therapists are professionals trained to deliver therapy where the emphasis is on improving occupational function and participation rather than treating symptoms, and uses a wide range of methods based on the needs of individuals. However, similar interventions may also be delivered by staff not trained as occupational therapists.

\section{Objectives}

To examine the effects of occupational therapy delivered by occupational therapists compared to occupational therapy delivered by any other person for people with schizophrenia.

\section{Search methods}

We searched the Cochrane Schizophrenia Group's Study-Based Register of Trials (including trial registers) on 4 November 2016 and 26 July 2018.

\section{Selection criteria}

All randomised controlled trials evaluating the functional or clinical outcomes of occupational therapy, or both, for people with schizophrenia delivered by occupational therapists compared with occupational therapy for people with schizophrenia delivered by anyone other than occupational therapists.

\section{Data collection and analysis}

Review authors independently inspected citations, selected studies, extracted data, and appraised study quality. 


\section{Main results}

The search yielded 1633 records. Of these, we retrieved 17 full-text reports (14 studies) for further scrutiny, which we subsequently excluded as they did not meet our inclusion criteria.

\section{Authors' conclusions}

Currently there are no randomised controlled trials comparing delivery of occupational therapy for people diagnosed with schizophrenia by occupational therapists with delivery of similar interventions by anyone other than occupational therapists. Research studies employing methodologically robust trial designs are needed to establish whether or not there are better outcomes for people with a diagnosis of schizophrenia with occupational therapy that is delivered by trained occupational therapists.

\section{PLAIN LANGUAGE SUMMARY}

\section{Occupational therapy for schizophrenia provided by occupational therapists compared to others}

\section{Review question}

Is there good-quality evidence that occupational therapy for people with a diagnosis of schizophrenia is more effective if it is provided by trained occupational therapists rather than anyone else?

\section{Background}

Schizophrenia is a serious mental health condition with a range of symptoms including hearing voices, unusual beliefs, paranoia, disorganised thinking or speech, and loss of motivation. In addition to people diagnosed with schizophrenia receiving medical treatments, services that support such individuals often provide further therapies such as occupational therapy. Occupational therapy aims to help improve the functioning and ability to take part in meaningful activities of people with schizophrenia, rather than focusing on reducing symptoms. Occupational therapy may be provided by specialist professionals trained as occupational therapists. However, similar therapy may be provided by other people who are not trained occupational therapists (e.g. doctors, nurses, other allied health professionals, or support staff). We wanted to find out whether there were any differences between the effectiveness of occupational therapy provided by occupational therapists and similar therapy provided by people not trained as occupational therapists.

\section{Searching for evidence}

On 26 July 2018 we ran an electronic search of the Cochrane Schizophrenia Group's specialised register of trials for clinical trials that allocated people with schizophrenia randomly to receive occupational therapy provided by an occupational therapist or receive occupational therapy provided by anyone else.

\section{Evidence found}

We identified 1633 potentially relevant records through our database searching, from which we obtained 17 full-text articles for closer inspection. We were unable to include any of these studies in the review and excluded a total of 14 studies.

\section{Conclusions}

At present there is no evidence from randomised controlled trials to permit a judgement as to whether occupational therapy for people with a diagnosis of schizophrenia is more effective if it is provided by trained occupational therapists compared to anyone else. Research is needed to answer this question and to help service providers plan future services that include occupational therapy for people with a diagnosis of schizophrenia. 


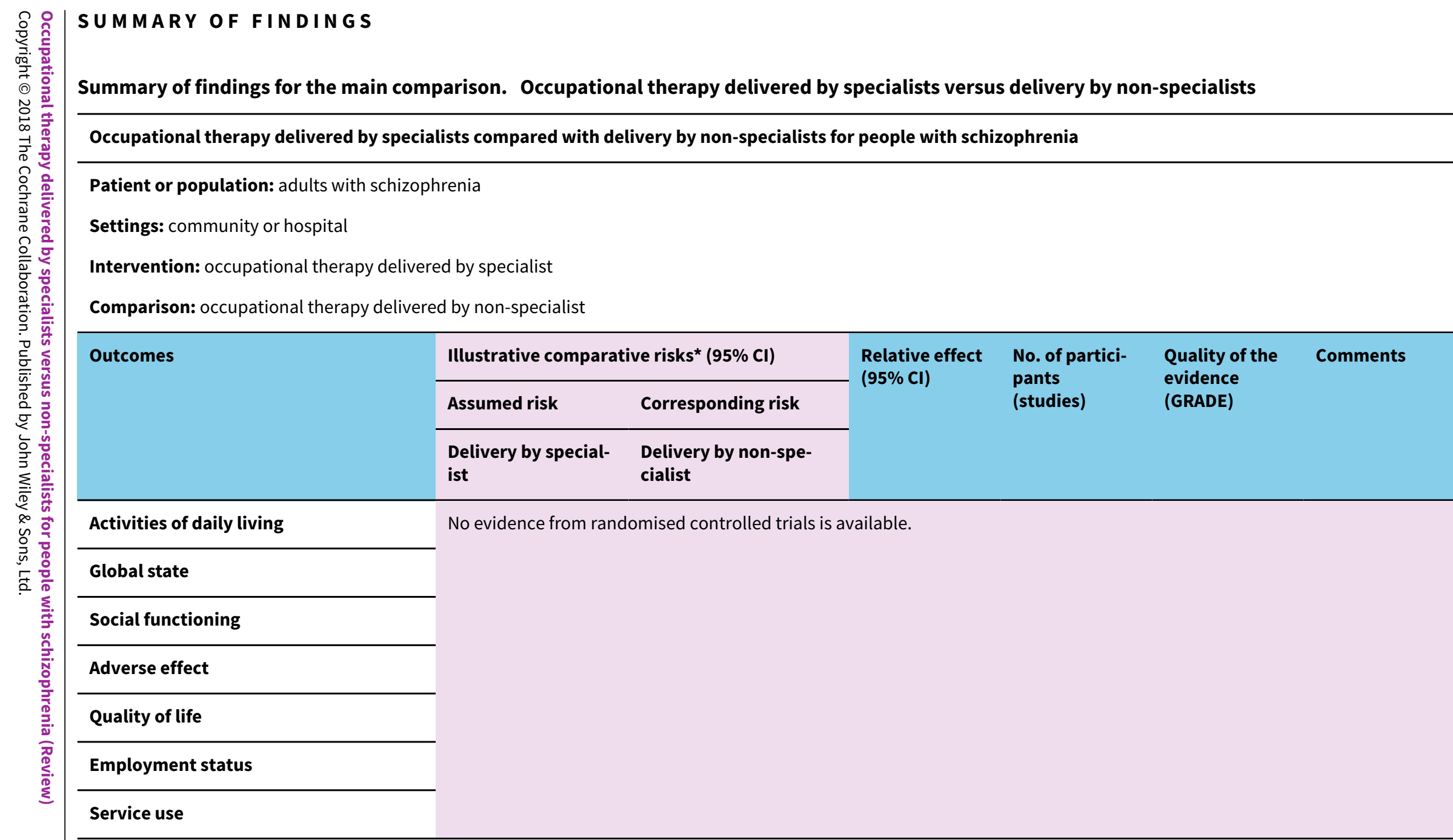

*The basis for the assumed risk (e.g. the median control group risk across studies) is provided in footnotes. The corresponding risk (and its $95 \%$ confidence interval) is based on the assumed risk in the comparison group and the relative effect of the intervention (and its $95 \% \mathrm{Cl}$ ).

Cl: confidence interval

GRADE Working Group grades of evidence

High quality: Further research is very unlikely to change our confidence in the estimate of effect.

Moderate quality: Further research is likely to have an important impact on our confidence in the estimate of effect and may change the estimate.

Low quality: Further research is very likely to have an important impact on our confidence in the estimate of effect and is likely to change the estimate. 


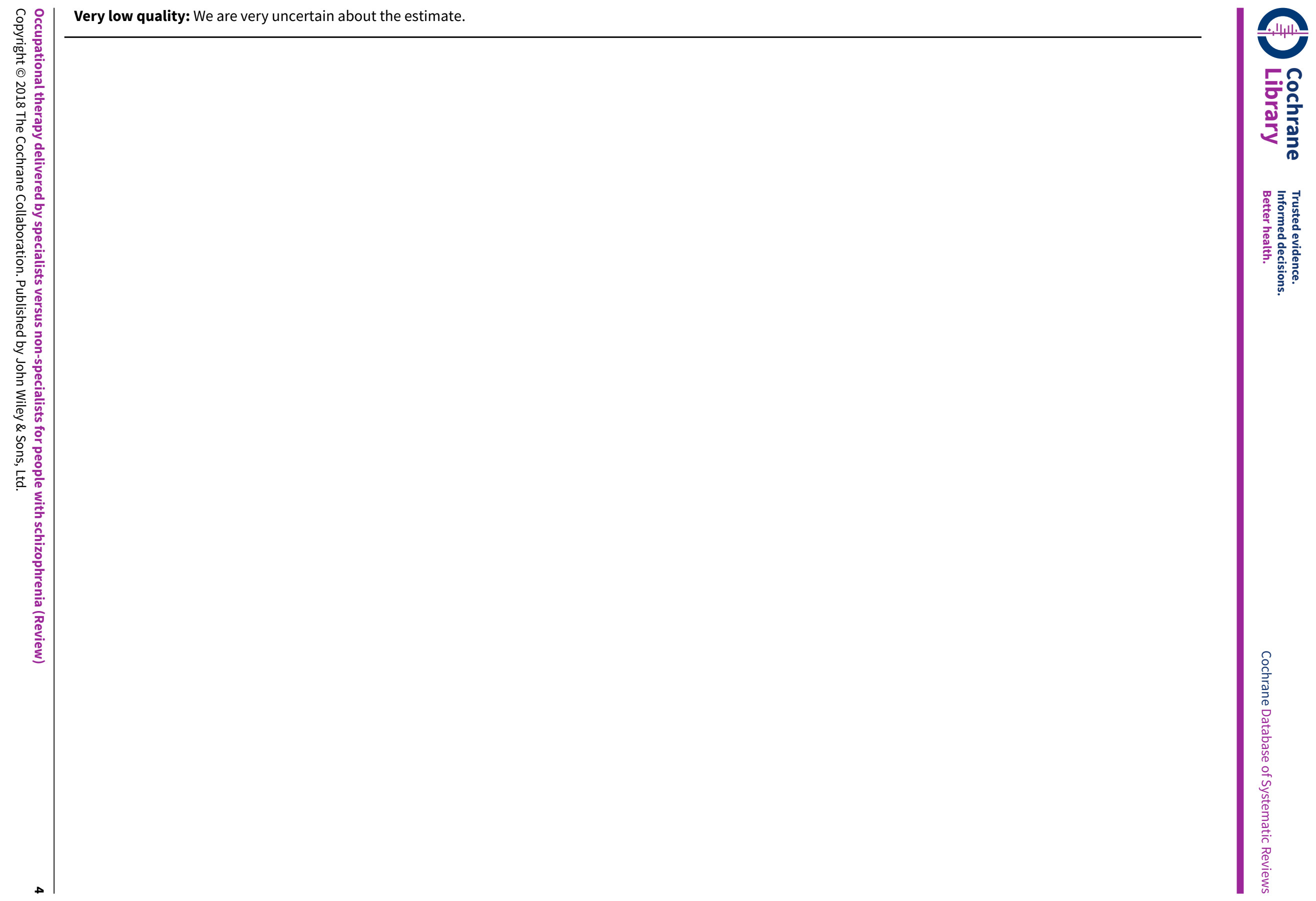




\section{B A C K G R O U N D}

\section{Description of the condition}

Schizophrenia is a severe mental illness that is characterised by positive symptoms, such as hallucinations and delusions; negative symptoms, such as catatonia, flattened affect, thought disorder (disrupted speech), and lack of motivation; and cognitive symptoms, such as problems with memory and attention (Carpenter 1994; Fioravanti 2005; NIMH 2014). Schizophrenia can occur as an isolated episode. However, for most people with schizophrenia it is a chronic illness characterised by a cycle of remission and relapse, which often leads to long-term disability (Bustillo 2000). It is among the top 15 medical conditions associated with impaired functioning (Murray 2013; NICE 2014). The first episode of schizophrenia most frequently occurs in males in their early $20 \mathrm{~s}$ and females in their late 20s. It is associated with impairment of both psychosocial and occupational functioning (APA 2013).

The World Health Organization (WHO) estimates that more than 21 million people worldwide are living with schizophrenia (WHO 2015). The median incidence of schizophrenia is estimated at 15.2 cases per 100,000 of the general population with lifetime prevalence estimated at 7.2 per 1000 of the general population (McGrath 2008). Prevalence is higher in males compared to females (rate ratio 1.4:1), and the mortality risk for people with schizophrenia is two to three times that of the general population, with an all-cause standardised mortality ratio of 2.6:1 (McGrath 2008).

A number of factors, including migrant status, urban living, and residence in high-income countries, are also associated with an increased risk of schizophrenia (McGrath 2008; McGrath 2009). The global burden of disease is substantial. Schizophrenia is defined as the most disabling condition in this disease classification group, and accounts for $7.4 \%$ of total disability-adjusted life years (DALYs) attributable to mental and substance use disorders. The peak burden occurs between 25 to 50 years of age (Whiteford 2013). It is estimated that only $21 \%$ of people of working age with schizophrenia are in paid employment (Marwaha 2007), with combined direct costs of treatment and indirect costs to society (e.g. unemployment, absenteeism, and premature mortality) in England during 2004/2005 of around GBP 6.7 billion (Mangalore 2007). The cost of schizophrenia is estimated at GBP 11.8 billion per year in England, with a public sector cost of GBP 7.2 billion (Andrews 2012). The costs arise from a range of factors, including inpatient time, loss of employment, disrupted education, homelessness, associated physical health problems, substance misuse, contact with the criminal justice system, and unpaid care provided by family members.

\section{Description of the intervention}

Occupational therapy is a complex intervention that incorporates the dynamic interchange of a range of personal and environmental factors (Creek 2005). While antipsychotic drugs are the mainstay of treatment for people with schizophrenia, these are often only part of a larger package of care that involves multiple healthcare professionals and therapies. Occupational therapists are a core member of multiprofessional teams that care for people with schizophrenia, and have unique skills in activity and occupational analysis that complement the skills of other members of the multiprofessional team (Creek 2005; WFOT 2010).
Occupational therapy is designed to support and enable continued participation in daily life through engagement in activities and occupations meaningful to the individual (WFOT 2010). Occupational therapists are uniquely trained to work across a broad range of physical, mental health, and social settings where the emphasis of therapy is on improving function rather than treating the symptoms of schizophrenia. Through modification of daily activities or the environment, or both, occupational therapists facilitate meaningful engagement in life activities (Creek 2003; WFOT 2010). Occupational therapy is not prescriptive, and a wide range of interventions are used when working with people, depending on their individual needs, preferences, and interests (Creek 2005; WFOT 2010). Common occupational therapy interventions include helping children with disabilities to participate fully in school and social situations, helping people recovering from injury to regain skills, and providing support for older adults who are experiencing physical and cognitive changes.

Schizophrenia impacts on a person's ability to participate in activities and engage with social roles (NICE 2014). Occupational therapists work in both hospital and community settings using a combination of individual and group interventions (Cook 2007; Smith 2014). The occupational therapist aims to use the activities that are important to the individual to help them increase skills that can help them live a fulfilling life (Urlic 2010).

\section{How the intervention might work}

People with schizophrenia can experience difficulty engaging in everyday life (Nagle 2002). This has been attributed to negative symptoms, Mairs 2004, and to symptom severity (Bejerholm 2004).

Occupational therapy interventions for people with long-term mental health issues such as schizophrenia aim to improve quality of life and social participation (Bryant 2014). This is achieved through adaptation of activities and environments important to the individual to enable skill development and building of their confidence in the execution of everyday tasks (Bryant 2014; Cook 2007; Smith 2014). This may include:

- practical self care;

- domestic skills, such as cooking and budgeting;

- work skills;

- leisure activities;

- development of social skills;

- carer support.

Occupational therapy focuses on occupations and personal strengths rather than problems, and thereby promotes the development of self determination, confidence, and understanding of health and well-being needs (COT 2006). Occupational therapists are trained to analyse, grade, and adapt occupations to suit personal circumstances and individual needs, and they actively involve people with the therapy within the framework of their own treatment and recovery journey. Occupational therapist-led interventions improve the quality of life and well-being for people with long-term mental health conditions such as schizophrenia (Aubin 1999). The development and maintenance of these skills has been shown to reduce readmission to hospital (Smith 2014). 


\section{Why it is important to do this review}

Currently there are no published formal evaluations of the evidence on the effectiveness of specialist-administered occupational therapy compared to occupational therapy delivered by other healthcare providers for people with schizophrenia. We aimed to evaluate the effectiveness of training specialised occupational therapists for enhancing the outcomes of occupational therapy. This will provide clinically useful information to enhance the quality of care among people with schizophrenia, to help clinicians in developing integrated care pathways, and to assist health policymakers in planning resource allocation.

\section{O B JE C T IVES}

To examine the effects of occupational therapy delivered by occupational therapists compared to occupational therapy delivered by any other person for people with schizophrenia. Our secondary objectives were to determine whether the response differs by specific type (e.g. hospital versus non-hospital setting), intensity (e.g. more therapist contact time or more frequent task repetition), or duration of occupational therapy.

\section{METHODS}

\section{Criteria for considering studies for this review}

\section{Types of studies}

We planned to include all relevant randomised controlled trials. If a trial was described as 'double-blind' but implied randomisation, we planned to include such trials in a sensitivity analysis (see the Sensitivity analysis). We planned to exclude quasi-randomised studies, such as those that allocated participants by alternate days of the week. Had we found studies where participants were given additional treatments alongside occupational therapy, we would only have included data if the adjunct treatment was evenly distributed between groups and only the occupational therapy was randomised.

\section{Types of participants}

We included people diagnosed with schizophrenia or related disorders, including schizophreniform disorder, schizoaffective disorder, and delusional disorder, by any means of diagnosis and irrespective of age, sex, or severity of illness. If trials included participants with a range of psychiatric diagnoses, we would only have included data reported separately for people with a diagnosis of schizophrenia.

We aimed to ensure that all information was as relevant to the current care of people diagnosed with schizophrenia as possible. We therefore proposed to clearly highlight the current clinical state (acute, early postacute, partial remission, remission), the stage (prodromal, first episode, early illness, persistent), and whether the included studies focused primarily on people with particular problems (e.g. negative symptoms, treatment-resistant illnesses).

\section{Types of interventions}

\section{Occupational therapy delivered by occupational therapists}

Study publications were unlikely to report the credentials of occupational therapists. We therefore defined an occupational therapist as anyone the study authors described as such.

\section{Occupational therapy delivered by anyone other than occupational therapists}

For example, doctors, nurses, allied health professionals, or support staff.

Where the included studies did not state who delivered the occupational therapy interventions, we planned to contact the study authors for clarification. We intended to exclude studies where we were unable to ascertain which professionals delivered the occupational therapy.

\section{Types of outcome measures}

We planned to divide outcomes into short-term (less than six months), medium-term (seven to 12 months), and long-term (more than one year) outcomes.

\section{Primary outcomes}

\section{Activities of daily living (ADL)}

For example, standard occupational therapy assessments, such as those based on the Model of Human Occupation (Kielhofner 2008) (such as the Model of Human Occupation Screening Tool (Parkinson 2006)), or Assessment of Motor and Process Skills (AMPS 2010), or the Canadian Occupational Performance Measure (Law 2005)

1.1 Clinically important change ADL (as defined by individual study)

\section{Global state}

2.1 Clinically important change global state (as defined by individual study)

\section{Adverse effect}

3.1 Any clinically important adverse effect (as defined by individual study)

\section{Secondary outcomes}

\section{Activities of daily living (ADL)}

1.1 Any change in ADL (as defined by individual study) 1.2 Average endpoint/change score ADL scale

\section{Global state}

2.2 Any change in global state (as defined by individual study)

2.3 Average endpoint/change score global state scale, e.g. the Brief Psychiatric Rating Scale (BPRS) (Overall 1988)

\section{Adverse effect/event}

3.1 Any specific effects (as defined by individual study) 3.2 Average endpoint/change score adverse effect scale 3.2 Death (suicide or natural cause)

\section{Quality of life}

4.1 Clinically important change in quality of life (as defined by individual study)

4.2 Any change in quality of life (as defined by individual study)

4.3 Average endpoint/change score quality of life scale, e.g. the EuroQoL EQ-5D score (EuroQol Group 1990)

\section{Social functioning}

5.1 Clinically important change in social functioning (as defined by individual study) 
5.2 Any change in social functioning (as defined by individual study)

5.3 Average endpoint/change score social functioning scale, e.g. the Social Functioning Scale or the Social Occupational Functioning Scale (Birchwood 1990; Saraswat 2006)

\section{Employment status}

Employment may be paid or unpaid, as defined by the individual study.

\subsection{Number of participants in employment}

\section{Mental state}

7.1 Clinically important change mental state (as defined by individual study)

7.2 Any change in mental state

7.3 Average endpoint/change score mental state scale, e.g. Scale for the Assessment of Negative Symptoms (Andreasen 1989)

\section{Service use}

8.1 Hospital admission

8.2 Length of stay

\section{Economic}

9.1 Direct cost of care

9.2 Indirect cost of care

\section{'Summary of findings' table}

We planned to use the GRADE approach to interpret findings of included studies (Schünemann 2011). We would have used GRADEpro to export data from this review to create 'Summary of findings' tables. These tables would have provided outcomespecific information concerning the overall quality of evidence from each included study in the comparison, the magnitude of the effect of the interventions examined, and the sum of available data on all outcomes rated as important to patient care and decision making. We aimed to include the following main outcomes in the 'Summary of findings' table.

- Activities of daily living: clinically important change (as defined by individual study)

- Global state: clinically important change in global state (as defined by individual study)

- Social functioning: clinically important change in social functioning (as defined by individual study)

- Adverse effect: any significant adverse effect

- Quality of life: clinically important change in quality of life (as defined by individual study)

- Employment status: number of participants in employment

- Service use: hospital admission

\section{Search methods for identification of studies}

\section{Electronic searches}

\section{Cochrane Schizophrenia Group's Study-Based Register of Trials}

On 4 November 2016 and 26 July 2018, the Information Specialist searched the register using the following search strategy, which was developed based on a literature review and in consultation with the review authors. $\left({ }^{\star}(\mathrm{CMOP}){ }^{\star} \mathrm{OR}{ }^{*}(\mathrm{COPM}){ }^{\star} \mathrm{OR}\right.$ * $(\mathrm{MOHO}){ }^{\star} \mathrm{OR}{ }^{*} \mathrm{CMOP}{ }^{*} \mathrm{OR}$ * $\mathrm{COPM}$ * OR *Domestic Skill* OR *Ergotherap* OR *KAWA Model* OR ${ }^{\star}$ Meaningful Activit ${ }^{\star} \mathrm{OR}^{*} \mathrm{MOHO}$ * $\mathrm{OR}^{*}{ }^{\star} \mathrm{MOHOST}^{\star} \mathrm{OR}^{\star}$ Occupation ${ }^{\star}$ OR ${ }^{\star}$ Purposeful Activit ${ }^{\star} \mathrm{OR}^{\star}{ }^{\star}$ Vocation ${ }^{\star} \mathrm{OR}^{*}{ }^{*}$ Volition ${ }^{\star}$ Questionnaire ${ }^{\star}$ $\mathrm{OR}^{*} \mathrm{VQ}{ }^{\star} \mathrm{OR}{ }^{*}$ Work Skill*) in Title OR Abstract Fields of REFERENCE OR ( ${ }^{\star}$ Ergotherapy ${ }^{\star}$ OR ${ }^{\star}$ Occupation ${ }^{\star}$ OR *Vocation ${ }^{\star}$ OR ( ${ }^{\star}$ Work ${ }^{\star}$ AND ${ }^{\star}$ Skill*)) in Interventions Field of STUDY

In such study-based register, searching the major concept retrieves all the synonyms and relevant studies because all the studies have already been organised based on their interventions and linked to the relevant topics (Shokraneh 2017; Shokraneh 2018).

This register is compiled by systematic searches of major resources (Allied and Complementary Medicine Database (AMED), BIOSIS, Cochrane Central Register of Controlled Trials (CENTRAL), Cumulative Index to Nursing and Allied Health Literature (CINAHL), ClinicalTrials.gov, Embase, MEDLINE, PsycINFO, PubMed, World Health Organization International Clinical Trials Registry Platform (WHO ICTRP)) and their monthly updates, ProQuest Dissertations and Theses $A \& I$ and its quarterly update, Chinese databases (Chinese Biomedical Literature (CBM), China National Knowledge Infrastructure (CNKI), and WANFANG) and their annual updates, handsearches, grey literature, and conference proceedings (see Group's website). There are no language, date, document type, or publication status limitations for inclusion of records into the register.

\section{Searching other resources}

\section{Reference searching}

We planned to inspect references of all included studies for further relevant studies.

\section{Personal contact}

We planned to contact the first author of each included study for information regarding unpublished trials. We intended to note the outcome of this contact in the included or awaiting assessment studies tables.

\section{Data collection and analysis}

\section{Selection of studies}

Two review authors (KM and GR) independently screened citations from the searches by title and abstract to identify articles that potentially met the inclusion criteria of the review. One review author (SS) independently re-inspected a random 20\% sample to ensure reliability of the review authors' assessments. Where disputes arose, we retrieved the full-text article(s) for further assessment. Two review authors (KM and GR) obtained and inspected the full-text articles of potentially relevant abstracts. SS also inspected the reports in order to ensure reliable selection. If we could not resolve disagreements by discussion, we planned to attempt to contact the study authors for clarification. We listed all excluded studies and their reasons for exclusion in the 'Characteristics of excluded studies' table. We constructed a PRISMA diagram to illustrate the study selection process. 


\section{Data extraction and management}

\section{Extraction}

Had we found studies suitable for inclusion, two review authors (KM and GR) would have extracted data from all included studies. In addition, to ensure reliability, SS would have independently extracted data from a random sample of these studies, comprising $10 \%$ of the total. Again, we would have discussed any disagreement, documented decisions, and, if necessary, contacted study authors for clarification. SS would have helped to resolve any remaining issues, and we would have documented these final decisions in the review text. We would have attempted to extract data presented only in graphs and figures whenever possible, but would have included data only if two review authors independently had the same result. We would have attempted to contact study authors through an open-ended request in order to obtain missing information or for clarification whenever necessary. For multicentre studies, where possible, we would have extracted data relevant to each component centre separately.

\section{Management}

\subsection{Forms}

Had we found studies suitable for inclusion, we would have extracted data on standardised data extraction forms.

\subsection{Scale-derived data}

We would have included continuous data from rating scales only if:

a) the psychometric properties of the measuring instrument have been described in a peer-reviewed journal (Marshall 2000);

b) the measuring instrument has not been written or modified by one of the trialists for that particular trial; and

c) the instrument should be a global assessment of an area of functioning and not subscores which are not, in themselves, validated or shown to be reliable. However, as an exception we would have included subscores from mental state scales measuring positive and negative symptoms of schizophrenia.

We realise that this is often not reported clearly, therefore we intended to note the instrument mode of completion in the Description of studies section of the review.

\subsection{Endpoint versus change data}

There are advantages in using both endpoint and change data. Change data can remove a component of between-person variability from the analysis. On the other hand, calculation of change needs two assessments (baseline and endpoint), which can be difficult in unstable and hard-to-measure conditions such as schizophrenia. We decided to primarily use endpoint data, and only use change data if the former were unavailable. Had we found studies suitable for inclusion, we would have combined endpoint and change data in the analysis, as we aimed to use mean difference (MD) values rather than standardised mean difference (SMD) values throughout (Deeks 2011).

\subsection{Skewed data}

Continuous data on clinical and social outcomes are often not normally distributed. To avoid the pitfall of applying parametric tests to non-parametric data, we planned to apply the following standards to all data before inclusion.

\section{For endpoint data $\mathbf{N}>\mathbf{2 0 0}$}

We would have entered data from trials with at least 200 participants because skewed data pose less of a problem in large studies.

\section{Change data}

We would have entered all change data because where continuous data scales include potential negative values (such as change data), it is difficult to identify whether the data are skewed.

\section{For endpoint data $\mathbf{N}<200$}

a) When a scale starts from the finite number zero, we planned to subtract the lowest possible value from the mean, and divide this by the standard deviation (SD). If this value was lower than one, it would strongly suggest a skew, and we would have excluded such data. If this ratio was higher than one but below two, it would suggest skew. We would have entered these data and tested whether their inclusion or exclusion would have changed the results substantially. Finally, if the ratio was larger than two, we would have included such data, because skew would be less likely (Altman 1996; Higgins 2011a).

b) If a scale started from a positive value (such as the Positive and Negative Syndrome Scale (PANSS) (Kay 1986), which can have values from 30 to 210), we would have modified the calculation described above to take the scale starting point into account. In these cases skew would have been considered to be present if 2 $\mathrm{SD}>$ (S - S min), where S was the mean score and 'S min' was the minimum score.

\subsection{Common measure}

To facilitate comparison between trials, we aimed to convert variables that could be reported in different metrics, such as days in hospital (mean days per year, per week, or per month) to a common metric (e.g. mean days per month).

\subsection{Conversion of continuous to binary}

If possible, we would have attempted to convert outcome measures to dichotomous data. This could have been done by identifying cut-off points on rating scales and dividing participants accordingly into 'clinically improved' or 'not clinically improved'. It is generally assumed that if there is a $50 \%$ reduction in a scale-derived score such as the Brief Psychiatric Rating Scale (BPRS), Overall 1962, or the PANSS, Kay 1986, this could be considered as a clinically significant response (Leucht 2005a; Leucht 2005b). If data based on these thresholds had been unavailable, we would have used the primary cut-off presented by the original study authors.

\subsection{Direction of graphs}

If possible, we would have entered data in such a way that the area to the left of the line of no effect indicated a favourable outcome for occupational therapists. Where keeping to this made it impossible to avoid outcome titles with clumsy double-negatives (e.g. 'not unimproved'), we would have reported data where the left of the line indicated an unfavourable outcome and noted this in the relevant graphs. 


\section{Assessment of risk of bias in included studies}

Had we found studies suitable for inclusion, two review authors (SS and KM) would have independently assessed the risk of bias in the included trials using the criteria described in the Cochrane Handbook for Systematic Reviews of Interventions (Higgins 2011b). This set of criteria is based on the evidence of associations between the overestimate of effect and high risk of bias of the article, such as sequence generation, allocation concealment, blinding, incomplete outcome data, and selective reporting.

In case of disagreement between review authors, we would have decided the final rating by consensus with the involvement of a third review author (SS). Where included trials provided inadequate details of randomisation and other characteristics of trials, we planned to contact the trial authors in order to obtain further information. We intended to report non-concurrence in quality assessment, and would have resolved by discussion any disputes regarding to which category a trial would have been allocated.

Had we found studies suitable for inclusion, we would have reported the results of the 'Risk of bias' assessment of the included trials within the review text and in the 'Summary of findings' table.

\section{Measures of treatment effect}

\section{Binary data}

For binary outcomes we would have calculated a standard estimation of the risk ratio (RR) and its 95\% confidence interval (Cl). It has been shown that RRs are more intuitive than odds ratios (ORs) (Boissel 1999), and that clinicians tend to interpret ORs as RRs (Deeks 2000). The number needed to treat for an additional beneficial outcome (NNTB)/number needed to treat for an additional harmful outcome (NNTH) statistic with its $\mathrm{Cls}$ is intuitively attractive to clinicians, but is problematic both in its accurate calculation in meta-analyses and interpretation (Hutton 2009). For binary data presented in the 'Summary of findings' table(s), we would have calculated illustrative comparative risks where possible.

\section{Continuous data}

For continuous outcomes we intended to estimate the MD between groups. We would prefer not to calculate effect size measures (SMD). However, had any included trials used scales of very considerable similarity, we would have assumed there was a small difference in measurement, and would have calculated effect size and transformed the effect back to the units of one or more of the specific instruments.

\section{Unit of analysis issues}

\section{Cluster-randomised trials}

Studies increasingly employ cluster randomisation (such as randomisation by clinician or practice), but analysis and pooling of clustered data poses problems. Firstly, study authors often fail to account for intraclass correlation in cluster-randomised studies, which leads to a 'unit of analysis' error whereby P values are spuriously low, Cls unduly narrow, and statistical significance overestimated (Divine 1992). This causes type I errors (Bland 1997; Gulliford 1999).

Had we found studies suitable for inclusion, where trial authors did not account for clustering in primary studies, we would have presented data in a table with an asterisk $\left(^{*}\right)$ to indicate the presence of a probable unit of analysis error. In subsequent versions of this review we will seek to contact first authors of included studies to obtain intraclass correlation coefficients (ICCS) for their clustered data and to adjust for this by using accepted methods (Gulliford 1999). Had we found studies suitable for inclusion, where clustering was incorporated into the analysis of primary studies, we would have presented these data as if from a non-cluster-randomised study but would have adjusted for the clustering effect.

We sought statistical advice and were advised that binary data as presented in a report should be divided by a 'design effect'. This is calculated using the mean number of participants per cluster $(\mathrm{m})$ and the ICC [Design effect $\left.=1+(m-1)^{\star} I C C\right]$ (Donner 2002). If the ICC was not reported, we would have assumed it to be 0.1 (Ukoumunne 1999).

Had we included cluster-randomised studies that had been appropriately analysed taking account of ICCs and relevant data documented in the report, synthesis with other studies would have been possible using the generic inverse variance technique.

\section{Cross-over trials}

A major concern of cross-over trials is the carry-over effect, which occurs if an effect (e.g. pharmacological, physiological, or psychological) of the treatment in the first phase is carried over to the second phase. As a consequence, on entry to the second phase the participants can differ systematically from their initial state despite a wash-out phase. For the same reason, cross-over trials are inappropriate if the condition of interest is unstable (Elbourne 2002). Cross-over study designs would be inappropriate for this intervention (occupational therapists), as it would not be possible to conceal the interventions or to avoid carry-over effects. Had we identified cross-over studies that met the inclusion criteria of this review, we would only have used data from the first phase of these studies in our analyses.

\section{Studies with multiple treatment groups}

Had we found studies suitable for inclusion, where a study involved more than two treatment arms, we would have presented the additional treatment arms in comparisons if relevant. If data were binary, we would have simply added and combined these data within the two-by-two table. If data were continuous, we would have combined data following the formula in the Cochrane Handbook for Systematic Reviews of Interventions (Higgins 2011a). If the additional treatment arms were irrelevant, we would not have use these data.

\section{Dealing with missing data}

\section{Overall loss of credibility}

At some degree of loss of follow-up data must lose credibility (Xia 2009). Should more than $50 \%$ of data have been unaccounted for regarding any given outcome, we would not have reproduced these data or used them within analyses. If, however, more than $50 \%$ of data in one study arm was lost, but the total loss was less than $50 \%$, we would have addressed this within the 'Summary of findings' table(s) by downgrading the quality of the evidence. Finally, if the loss was between $25 \%$ to $50 \%$ in total, we would also have downgraded the quality of the evidence. 


\section{Binary}

In the case where attrition for a binary outcome is between $0 \%$ and $50 \%$ and where the trial authors did not clearly describe these data, we would have presented data on an intention-to-treat (ITT) basis. We would have assumed that those participants that left the study early had the same rates of negative outcome as those who completed the study, with the exception of the outcomes of death and adverse effects. For these outcomes, we would have used the rate of those who stayed in the study - in that particular trial arm - for those who did not. We planned to undertake a sensitivity analysis to test how prone the primary outcomes were to change when we compared data only from people who completed the study to that point to the ITT analysis using the above assumptions.

\section{Continuous}

\subsection{Attrition}

In the case where attrition for a continuous outcome is between $0 \%$ and $50 \%$, and the trial(s) only reported data from people who complete the study to that point, we planned to use these data.

\subsection{Standard deviations}

Had any included trials not reported SD values, we would have first attempted to obtain the missing values from the trial authors. If these data were unavailable, where there were missing measures of variance for continuous data, but an exact standard error (SE) and $\mathrm{Cls}$ were available for group means, and either the $\mathrm{P}$ value or ' $\mathrm{t}$ ' value were available for the MDs, we would have calculated them according to the Cochrane Handbook for Systematic Reviews of Interventions (Higgins 2011a). When the trial authors only reported the SE values, we would have calculated SD values using the formula SD $=\mathrm{SE}$ * square root (n). The Cochrane Handbook for Systematic Reviews of Interventions presents detailed formulas for estimating SDs from $\mathrm{P}$ values, t or $\mathrm{F}$ values, Cls, ranges, or other statistics (Higgins 2011a). If these formulas did not apply, we would have calculated the SDs according to a validated imputation method which is based on the SDs of the other included studies (Furukawa 2006). Although some of these imputation strategies can introduce error, the alternative would have been to exclude a given study's outcome and thus to lose information. Nevertheless, we planned to examine the validity of the imputations in a sensitivity analysis by excluding imputed values.

\subsection{Assumptions about participants who left the trials early or were lost to follow-up}

Various methods are available to account for participants who leave a trial early or who are lost to follow-up. Some trials just present the results of study completers, while other studies use the method of last observation carried forward (LOCF). More recently, methods such as multiple imputation or mixed-effects models for repeated measurements (MMRM) have become more of a standard. While the latter methods seem to be somewhat better than LOCF (Leon 2006), we feel that the high percentage of participants leaving the studies early and the differences in the reasons for leaving the studies early between groups is often the core problem in randomised trials of people with schizophrenia. We therefore decided not to exclude studies based on the statistical approach the trial authors used. However, had we found studies suitable for inclusion, we would preferably have used the more sophisticated approaches, for example we would have preferred MMRM or multiple imputation to LOCF, and would have presented completer analyses only if no kind of ITT data were available. Moreover, we would have addressed this issue in the 'incomplete outcome data' item of the 'Risk of bias' tool.

\section{Assessment of heterogeneity}

\section{Clinical heterogeneity}

Had we found studies suitable for inclusion, we would have considered all included studies initially, without seeing comparison data, to judge clinical heterogeneity. We intended to inspect all studies for clearly outlying participants or situations that we had not predicted would arise. Had we identified such situations or participant groups, we would have fully discussed them.

\section{Methodological heterogeneity}

Had we found studies suitable for inclusion, we would have considered all included studies initially, without seeing comparison data, to judge methodological heterogeneity. We intended to inspect all studies for clearly outlying methods that we had not predicted would arise. Had we identified such methodological outliers, we would have fully discussed them.

\section{Statistical heterogeneity}

\subsection{Visual inspection}

Had we found studies suitable for inclusion, we would have visually inspected graphs to investigate the possibility of statistical heterogeneity.

\subsection{Employing the $I^{2}$ statistic}

Had we found studies suitable for inclusion, we would have investigated heterogeneity between studies by considering the $1^{2}$ statistic method alongside the $\mathrm{Chi}^{2}$ test $\mathrm{P}$ value. The $\mathrm{I}^{2}$ statistic provides an estimate of the percentage of inconsistency thought to be due to chance (Higgins 2003). The importance of the observed value of the $\mathrm{I}^{2}$ statistic depends on the magnitude and the direction of effects, and the strength of the evidence for heterogeneity (e.g. P value from $\mathrm{Chi}^{2}$ test, or a $\mathrm{Cl}$ for the $\mathrm{I}^{2}$ statistic value). We would have interpreted an 12 statistic estimate greater than or equal to around $50 \%$ accompanied by a statistically significant $\mathrm{Chi}^{2}$ test value as evidence of substantial levels of heterogeneity, per Chapter 9 of the Cochrane Handbook for Systematic Reviews of Interventions (Deeks 2011). Had we observed substantial levels of heterogeneity in the primary outcome, we would have explored the reasons for this (see Subgroup analysis and investigation of heterogeneity).

\section{Assessment of reporting biases}

\section{Protocol versus full study}

Reporting biases arise when the dissemination of research findings is influenced by the nature and direction of results. These are described in Chapter 10 of the Cochrane Handbook for Systematic Reviews of Interventions (Sterne 2011). We intended to locate protocols of included studies and, where available, to compare the outcomes in the protocol with those in the published report. If the protocol was not available, we intended to compare outcomes listed in the methods section of the trial report with those reported in the results.

\section{Funnel plot}

Again, reporting biases arise when the dissemination of research findings is influenced by the nature and direction of results (Egger 
1997). We are aware that funnel plots may be useful in investigating reporting biases but are of limited power to detect small-study effects. We would not have used funnel plots for outcomes for which there were 10 or fewer included studies, or where all studies were of similar size. In other cases, where funnel plots were possible, we would have sought statistical advice on their interpretation.

\section{Data synthesis}

We understand that there is no clear consensus on the use of fixed-effect or random-effects models. The random-effects method incorporates an assumption that the different studies are estimating different, yet related, intervention effects. This often seems to be true to us, and the random-effects model takes into account differences between studies even if there is no statistically significant heterogeneity. There is, however, a disadvantage to the random-effects model, in that it places added weight on small studies, which are often the most biased ones. Depending on the direction of effect, these studies can either inflate or deflate the effect size. Occupational therapists deliver therapy that is diverse in nature, therefore we made an a priori assumption that any included studies would estimate different but related effects. Had we included any studies in the review, we would have used a random-effects model for all analyses.

\section{Subgroup analysis and investigation of heterogeneity}

\section{Subgroup analyses}

\subsection{Primary outcomes}

For the primary outcomes we intended to determine whether the response to therapy varied according to the following subgroups:

- duration of therapy: short- (up to eight weeks) versus long-term;

- specific type: hospital versus non-hospital setting;

- intensity: more therapist contact time or more frequent task repetition (intensive programme).

\subsection{Clinical state, stage, or problem}

We proposed to undertake this review and provide an overview of the effects of occupational therapy delivered by occupational therapists for people with schizophrenia in general. However, if the included trials reported data for subgroups of people in the same clinical state, stage, and with similar problems, we would also have reported these for the primary outcomes.

\section{Investigation of heterogeneity}

Had we found studies suitable for inclusion, we would have reported whether inconsistency was high. Firstly, we would have investigated whether data were entered correctly. Secondly, if data were correct we would have visually inspected the graph and successively removed studies that appeared distinct from the remainder to see if homogeneity was restored. For this Cochrane Review, we decided that should this have occurred with data contributing to the summary findings of no more than around $10 \%$ of the total weighting, we would have presented such data. If not, we would not have pooled data and would have discussed any relevant issues. We know of no supporting research for this $10 \%$ cutoff, but we are investigating the use of prediction intervals as an alternative to this unsatisfactory state.

Had we found studies suitable for inclusion, when unanticipated clinical or methodological heterogeneity was obvious, we would have simply stated hypotheses regarding this for future reviews or future versions of this review. We did not anticipate undertaking analyses relating to such studies.

\section{Sensitivity analysis}

\section{Implication of randomisation}

Had we found studies suitable for inclusion, we aimed to include trials in a sensitivity analysis if the trial authors described them in some way as to imply randomisation. For the primary outcomes we would have included these studies. If their inclusion did not result in a substantive difference, they would have remained in the analyses. If their inclusion did result in important clinically significant but not necessarily statistically significant differences, we would not have added the data from these lower-quality studies to the results of the better-quality trials, but would have presented such data within a subcategory.

\section{Assumptions for lost binary data}

Had we found studies suitable for inclusion, where we had to make assumptions regarding participants lost to follow-up (see Dealing with missing data), we would have compared the findings of the primary outcomes when we used our assumption(s) and when we used data only from participants who completed the study to that point. Had there been a substantial difference, we would have reported the results and discussed them but would have continued to employ our assumption.

Had we needed to make assumptions regarding missing SD data (see Dealing with missing data), we would have compared the findings of the primary outcomes when we used our assumption(s) and when we used data only from participants who completed the study to that point. We would have undertaken a sensitivity analysis to test how prone the results were to change when we compared completer-only data to the imputed data using the above assumption. Had there been a substantial difference, we would have reported the results and discussed them but would have continued to employ our assumption.

\section{Risk of bias}

Had we found studies suitable for inclusion, for the primary outcomes, we would have analysed the effects of excluding trials that we judged to be at high risk of bias across one or more of the domains of randomisation (implied as randomised with no further details available, allocation concealment, blinding, and outcome reporting). If the exclusion of trials at high risk of bias did not substantially alter the direction of effect or the precision of the effect estimates, we would have included the data from these trials in the analysis.

\section{Imputed values}

Had we found studies suitable for inclusion, for the primary outcomes, we would have undertaken a sensitivity analysis to assess the effects of including data from trials where we used imputed values for the ICC in calculating the design effect in clusterrandomised trials.

Had we noted substantial differences in the direction or precision of effect estimates in any of the sensitivity analyses listed above, we would not have pooled data from the excluded trials with the 
other trials that contributed to the given outcome, but would have presented them separately.

\section{Fixed-effect and random-effects models}

Had we found studies suitable for inclusion, we would have synthesised all data using a random-effects model. However, we would also have synthesised data for the primary outcomes using a fixed-effect model to evaluate whether this altered the significance of the results.

\section{RE S U L T S}

\section{Description of studies}

Results of the search

A flow diagram of the study selection process is shown in Figure 1. 
Figure 1. Study flow diagram for 2016 and 2018 searches.

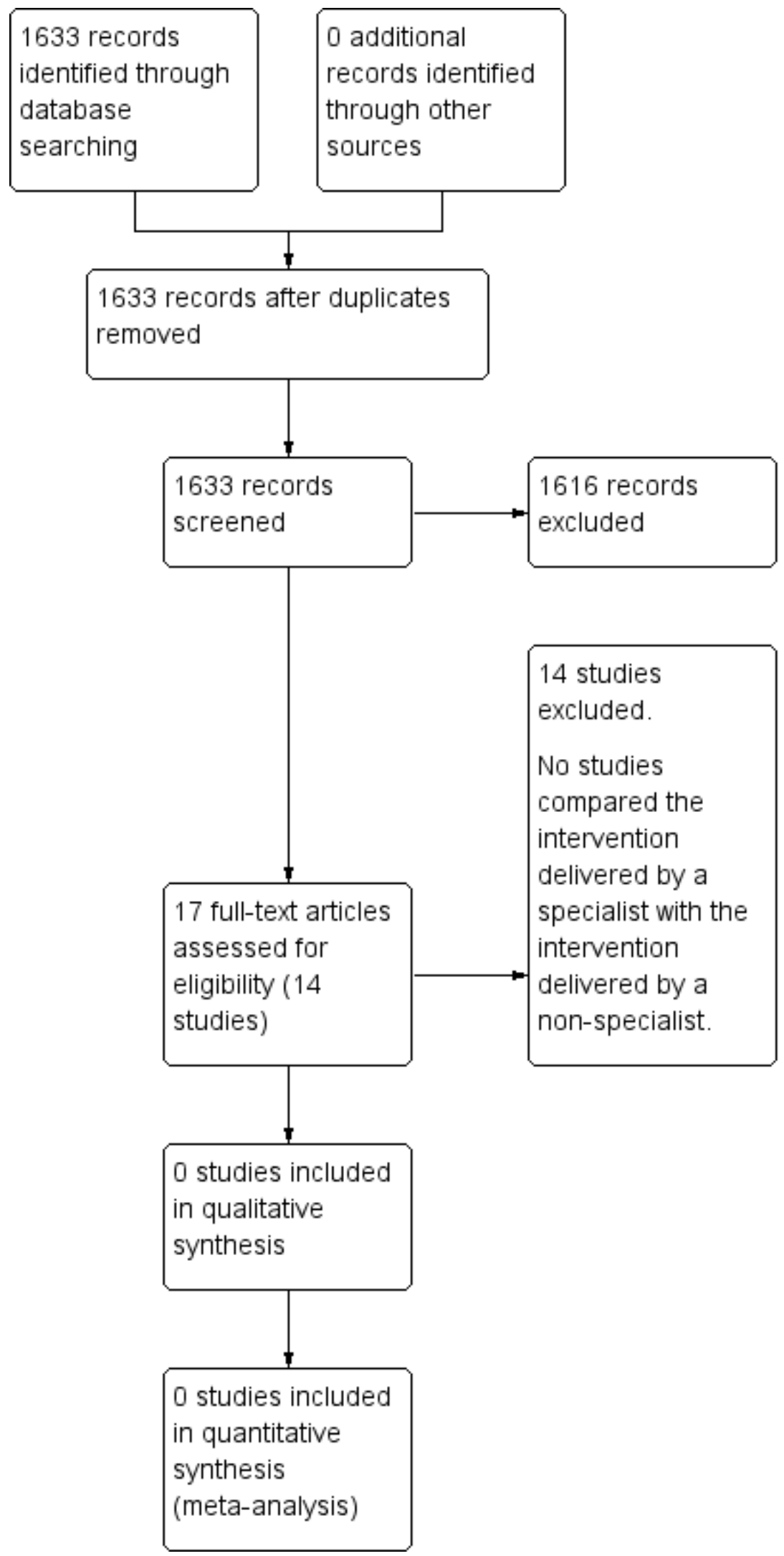


After removal of duplicates, review authors $\mathrm{KM}$ and $\mathrm{GR}$ independently screened the titles and abstracts of 1633 records for eligibility. Sai Zhao (see Acknowledgements) kindly screened the Chinese studies. Review authors KM, GR, and SS independently obtained and closely inspected the full texts of 17 records (referring to 14 studies). We did not identify any studies for inclusion in the review.

\section{Included studies}

None of the reports retrieved met the inclusion criteria for this review (see Criteria for considering studies for this review).

\section{Excluded studies}

We excluded all 14 studies found in the search. None of these studies compared occupational therapy delivered by occupational therapists with occupational therapy delivered by non-specialists, therefore they did not meet our inclusion criteria. See Characteristics of excluded studies for further details.

\section{Ongoing studies}

We are unaware of any ongoing studies.

\section{Studies awaiting assessment}

No studies are awaiting assessment.

\section{Risk of bias in included studies}

As no studies met the inclusion criteria (Criteria for considering studies for this review), it was not possible to assess risk of bias.

\section{Effects of interventions}

See: Summary of findings for the main comparison Occupational therapy delivered by specialists versus delivery by non-specialists

Due to the absence of data it was not possible to report on the effects of delivering occupational therapy by specialists for people with schizophrenia.

\section{DISCUSSION}

\section{Summary of main results}

This review aimed to compare the impact of occupational therapy delivered by occupational therapists with occupational therapy delivered by anyone other than occupational therapists for people with schizophrenia. However, despite an extensive search, we did not identify any studies for inclusion in the review (Criteria for considering studies for this review).

\section{Overall completeness and applicability of evidence}

We were unable to assess completeness and applicability of evidence as no studies were included in the review.

\section{Quality of the evidence}

We were unable to assess quality of the evidence as no studies were included in the review.

\section{Potential biases in the review process}

We consider our search to be rigorous and comprehensive. At each stage the review authors independently applied the inclusion criteria before comparing results. Reliability checking was carried out at screening stage.

\section{Agreements and disagreements with other studies or reviews}

There are currently no reviews on the Cochrane Library that evaluate the efficacy of occupational therapists delivering interventions in other clinical conditions, and similarly no reviews evaluating the clinical and economic impact of occupational therapy for people with schizophrenia. The 2015 European guideline on psychosocial therapies in severe mental illness included a review of the evidence for occupational therapy (Guhne 2015). The review identified very few randomised controlled trials of occupational therapy and concluded that the effects of occupational therapy as a mental health intervention have not been systematically evaluated. These findings are consistent with a related review assessing the impact of life skills for people with chronic mental illness, which based on a small number of low-quality studies found no benefit from the intervention (Tungpunkom 2012). A review of social skills programmes reported evidence of a benefit in social functioning, relapse and rehospitalisation rates, mental state, and quality of life, but was again based on a small number of poor-quality trials (Almerie 2015). Whilst we identified no studies that met the inclusion criteria for this review, the search did identify a number of randomised trials evaluating the impact of occupational therapy that would concur with the need for a systematic review, for example Campbell 1983; Du 2001; Gao 2004; Xiaoping 2014; Killaspy 2013; Debin 2015. We are also aware of other work evaluating the effectiveness and costeffectiveness of occupational therapy for people with psychotic conditions (Cook 2007; Cook 2009). These studies are evidence of emerging research within this field.

\section{AUTHORS' CONCLUSIONS}

\section{Implications for practice}

\section{For people with schizophrenia}

Research does suggest that occupational therapy can be beneficial for people diagnosed with schizophrenia (e.g. Bejerholm 2004; Smith 2014), but there is currently no evidence from randomised controlled trials to determine whether it is more effective when delivered by occupational therapists compared to non-specialists. People with schizophrenia, if offered occupational therapy by nonspecialists, need not feel the service they are getting is inferior to specialist-provided approaches.

\section{For clinicians}

Whether the occupational therapy is delivered by qualified occupational therapists or by non-specialist staff is important for occupational therapists. Clinicians need to be aware of the lack of evidence in this area and the need for further research to develop the evidence base and reduce uncertainties around the best way of delivering occupational therapy for people diagnosed with schizophrenia. In the interim, occupational therapy seems beneficial for people with schizophrenia and therapeutic strategy should therefore continue on the basis of best practice. However, in times of austerity, when resources are limited and waiting lists long, the only equitable - and therefore ethical - way of providing care is to use locally available resources, irrespective of whether they are specialist or non-specialist staff. 


\section{For policymakers}

Cook 2009 recommends occupational therapy as an effective intervention for people diagnosed with schizophrenia, but there is little evidence-based guidance on who should deliver the therapy. To optimise therapy and maximise any benefits, means of delivery (frequency, by whom/what) need to be evaluated, with a focus not only on clinical effects but also cost/benefit. This would assist in the planning of how to structure services and allocate resources, particularly in settings where access to occupational therapy is limited (e.g.low income countries).

\section{Implications for research}

\section{Reviews}

The excluded studies illustrate that it is possible to evaluate many aspects of occupational therapy for people with schizophrenia. Some of the comparisons outlined in Table 1 may fit into already existing reviews such as the life skills review (Tungpunkom 2012), but we believe there are many other reviews to be completed in this area.

\section{Trials}

Research is urgently required to determine whether occupational therapy delivered by specialists is as effective as occupational therapy delivered by non-specialists. With the high costs of therapy and the need to help patients remain independent and out of long-term care, it is essential that future policy is informed by the evidence base. This will help to determine who is best placed to deliver therapy with maximum cost-effective benefit for both patients and service providers. However, it is also essential to establish the effectiveness and cost-effectiveness of occupational therapy as an intervention in schizophrenia, particularly as evidence is available for systematic review on comparisons between occupational therapy and a) no occupational therapy or b) other types of activity-based therapy. This would help to determine which types of occupational therapy are the most effective, taking account of clinical variation and different settings.

Future trials are required to establish effects for important outcomes including the impact on activities of daily living, hospital readmissions, and the economic costs of care. Trials should also take account of the variability in methods of delivery (individual versus group), participants (adults versus children), and setting (hospital versus community), aiming to establish comparative effectiveness with maximum operational validity by evaluating the impact of the type of therapist in the context of usual care. See Table 2 for further details on the design of potential future trials.

\section{ACKNOWLEDGEMENTS}

The Cochrane Schizophrenia Group Editorial Base in Nottingham, UK produces and maintains standard text for use in the Methods section of their reviews. We have used this text as the basis for what appears here and have adapted it as required.

The Information Specialist of the Cochrane Schizophrenia Group and the contact author of this review developed the search term.

We would also like to thank Sai Zhao from Systematic Review Solutions Ltd, Yantai, China, for assisting with the translation of papers written in the Chinese language during the screening process.

We would also like to thank and acknowledge Sally Walsh and Tarek Turk for peer reviewing the protocol and the review. 


\section{REFERE N CE S}

\section{References to studies excluded from this review}

Anqiong 2010 \{published data only\}

An Qiong, Li Yulan. Analysis of the effect of entertainment therapy on elderly schizophrenia [工娱治疗对老年精神分裂 症的康复效果分析]. Chinese Journal of Practical Medicine 2010;5(27):237-8.

\section{Campbell 1983 \{published data only\}}

Campbell A, McCreadie RG. Occupational therapy is effective for chronic schizophrenic day-patients. Occupational Therapy 1983;2281:327-8.

\section{Chan 2007 \{published data only\}}

Chan SHW, Lee SWK, Chan IWM. TRIP: a psycho-educational programme in Hong Kong for people with schizophrenia. Occupational Therapy International 2007;14(2):86-98.

\section{Debin 2015 \{published data only\}}

Debin Z, Jia L, Xianglai L, Ranran S. A randomized controlled study of occupational therapy on cognitive function in hospitalized schizophrenic patients. Chinese Journal of Tropical Medicine 2015;15(4):506-8.

\section{Du 2001 \{published data only\}}

Du HQ, Chen YM, Yu L. A controlled study of the negative symptoms of schizophrenia treated by sulpiride combined with occupational and recreational therapy. \#\#\#\#\# [Shandong Psychiatry] 2001;14(1):40-2.

\section{Gao 2004 \{published data only\}}

Gao Y, Wang QY, Zhang XQ, Lai MD. Effect of occupational therapy on self-rated health measurement in schizophrenic patients. Chinese Journal of Clinical Rehabilitation 2004;8(18):3468-9.

\section{Guofu 2013 \{published data only\}}

Guofu Z. Effects of training methods based on the theory of human function on rehabilitation of schizophrenic patients. Journal of Practical Medicine 2013;29(19):3160-2.

\section{Hoshii 2013 \{published data only\}}

Hoshii J, Yotsumoto K, Tatsumi E, Tanaka C, Mori T, Hashimoto T. Subject-chosen activities in occupational therapy for the improvement of psychiatric symptoms of inpatients with chronic schizophrenia: a controlled trial. Clinical Rehabilitation 2013;27(7):638-45

\section{Killaspy 2013 \{published data only\}}

ISRCTN25898179. Rehabilitation Effectiveness and Activities for Life (REAL): a multicentre study of rehabilitation services and the efficacy of promoting activities for people with severe mental health problems. www.isrctn.com/ISRCTN25898179 (Date assigned 23 April 2010).

Killaspy H. Rehabilitation Effectiveness and Activities for Life (REAL): RCT phase. public.ukcrn.org.uk (Date accessed: 17 November 2011).
Killaspy H, Cook S, Mundy T, Craig T, Holloway F, Leavey G, et al. Study protocol: cluster randomised controlled trial to assess the clinical and cost effectiveness of a staff training intervention in inpatient mental health rehabilitation units in increasing service users' engagement in activities. BMC Psychiatry 2013;13:216.

* Killaspy H, Marston L, Green N, Harrison I, Lean M, Cook S, et al. Clinical effectiveness of a staff training intervention in mental health inpatient rehabilitation units designed to increase patients' engagement in activities (the Rehabilitation Effectiveness for Activities for Life (REAL) study): singleblind, cluster-randomised controlled trial. Lancet Psychiatry 2015;2(1):38-48

\section{Ruiling 2015 \{published data only\}}

Ruiling Y. Rehabilitation of inpatients with chronic schizophrenia. Chinese Journal of Trauma and Disability Medicine 2015;23(10):147-8.

\section{Xiaojing 2012 \{published data only\}}

Xiao-jing G, Ying-qiang Y, Da-guang Z, Jiu-ying W, Shu-hua W, Lu C. A preliminary study on the rehabilitation effect of occupational rehabilitation skill training on social function of schizophrenic patients. Chinese Medical Association 10th National Symposium on Psychiatry. 2012.

Xiaoping 2014 \{published data only\}

Xiaoping H, Fujun W, Mian S. Effects of occupational therapy on the improvement of negative symptoms in patients with chronic schizophrenia. Chinese Journal of Public Health Medicine 2014;26(22):29-31.

\section{Xiong 2013 \{published data only\}}

Xiong C, Bin L, Weixia L. Comparison of the effects of two kinds of entertainment therapy on the rehabilitation of rural schizophrenia in chronic patients. Journal of Medical Theory and Practice 2013;26(3):399-401.

\section{Yanyan 2016 \{published data only\}}

Yanyan Y, Fuyin Y, Wei T, Feixue Z, Yuexiao R, Liandan H. Evaluation of three-stage rehabilitation in hospitalized schizophrenic patients. Zhejiang Preventive Medicine 2016;28(01):43-4.

\section{Additional references}

Almerie 2015

Almerie MQ, Okba Al Marhi M, Jawoosh M, Alsabbagh M, Matar HE, Maayan N, et al. Social skills programmes for schizophrenia. Cochrane Database of Systematic Reviews 2015, Issue 6. [DOI: 10.1002/14651858.CD009006.pub2]

\section{Altman 1996}

Altman DG, Bland JM. Detecting skewness from summary information. BMJ 1996;313(7066):1200. 


\section{AMPS 2010}

Fisher AG, Jones KB. Assessment of Motor and Process Skills. Vol. 1: Development, Standardization, and Administration Manual. 7th Edition. Vol. 1, Fort Collins: Three Star Press, 2010.

\section{Andreasen 1989}

Andreasen NC. Modified Scale for the Assessment of Negative Symptoms (SANS). British Journal of Psychiatry 1989;155(7):53-8.

\section{Andrews 2012}

Andrews A, Knapp M, McCrone P, Parsonage M, Trachtenberg M. Effective interventions in schizophrenia. The economic case. A report prepared for the Schizophrenia Commission. Personal Social Services Research Unit, London School of Economics and Political Science. www.lse.ac.uk/LSEHealthAndSocialCare/pdf/ LSE-economic-report-FINAL-12-Nov.pdf. London, UK, (accessed 12 August 2016).

\section{APA 2013}

American Psychiatric Association. Schizophrenia and Other Psychotic Disorders. Diagnostic and Statistical Manual of Mental Disorders (DSM-V). 5th Edition. Washington, DC: American Psychiatric Association, 2013.

\section{Aubin 1999}

Aubin G, Hachey R, Mercier C. Meaning of daily activities and subjective quality of life in people with severe mental illness. Scandinavian Journal of Occupational Therapy 1999;6(2):53-62.

\section{Bejerholm 2004}

Bejerholm U, Eklund M. Time use and occupational performance among persons with schizophrenia. Occupational Therapy in Mental Health 2004;20(1):27-47.

\section{Birchwood 1990}

Birchwood M, Smith J, Cochrane R, Wetton S, Copestake S. The Social Functioning Scale - the development and validation of a new scale of social adjustment for use in the family intervention programme with schizophrenic patients. British Journal of Psychiatry 1990;157(6):853-9.

\section{Bland 1997}

Bland JM, Kerry SM. Statistics notes. Trials randomised in clusters. BMJ 1997;315(7108):600.

\section{Boissel 1999}

Boissel JP, Cucherat M, Li W, Chatellier G, Gueyffier F, Buyse $M$, et al. The problem of therapeutic efficacy indices. 3. Comparison of the indices and their use [Aperçu sur la problématique des indices d'efficacité thérapeutique, 3: comparaison des indices et utilisation. Groupe d'Etude des Indices D'Efficacité]. Therapie 1999;54(4):405-11. [PUBMED: 10667106]

\section{Boutron 2008}

Boutron I, Moher D, Altman DG, Schulz K, Ravaud P, for the CONSORT group. Extending the CONSORT Statement to randomized trials of nonpharmacologic treatment: explanation and elaboration. Annals of Internal Medicine 2008;148(4):295-309. [PUBMED: 18283207]

\section{Bryant 2014}

Bryant W, Fieldhouse J, Bannigan K. Creek's Occupational Therapy and Mental Health. 5th Edition. London: Churchill Livingstone, 2014.

\section{Bustillo 2000}

Bustillo J, Keith SJ, Lauriello J. Kaplan and Sadock's Comprehensive Book of Psychiatry. Philadelphia: Lippincott Williams \& Wilkins, 2000.

\section{Carpenter 1994}

Carpenter WT Jr, Buchanan RW. Schizophrenia. New England Journal of Medicine 1994;330(10):681-90.

\section{Chan 2013}

Chan A, Tetzlaff JM, Altman DG, Laupacis A, Gøtzsche, PC, Krleža-Jerić K, et al. SPIRIT 2013 Statement: defining standard protocol items for clinical trials. Annals of Internal Medicine 2013;158(3):200-7. [DOI: 10.7326/0003-4819-158-3-201302050-00583]

\section{Cook 2007}

Cook S, Birrell M. Defining an occupational therapy intervention for people with psychosis. British Journal of Occupational Therapy 2007;70(3):96-106.

\section{Cook 2009}

Cook S, Chambers E, Coleman JH. Occupational therapy for people with psychotic conditions in community settings: a pilot randomized controlled trial. Clinical Rehabilitation 2009;23(1):40-52.

\section{COT 2006}

College of Occupational Therapists. Recovering ordinary lives. The strategy for occupational therapy in mental health services 2007-2017. A vision for the next ten years. www.cot.co.uk/sites/ default/files/publications/public/ROL-Vision-2010.pdf (accessed 12 August 2016).

\section{Creek 2003}

Creek J. Occupational therapy defined as a complex intervention. www.cot.co.uk/publication/publications/ occupational-therapy-defined-complex-intervention. London: College of Occupational Therapists, (accessed 12 August 2016).

\section{Creek 2005}

Creek J, Ilott I, Cook S, Munday C. Valuing occupational therapy as a complex intervention. British Journal of Occupational Therapy 2005;68(6):281-4.

\section{Deeks 2000}

Deeks J. Issues in the selection for meta-analyses of binary data. The 8th International Cochrane Colloquium; 2000 Oct 25-28; Cape Town. Cape Town: The Cochrane Collaboration, 2000.

\section{Deeks 2011}

Deeks JJ, Higgins JPT, Altman DG (editors). Chapter 9: Analysing data and undertaking meta-analyses. In: Higgins JPT, Green $\mathrm{S}$ (editors). Cochrane Handbook for Systematic Reviews of 
Interventions Version 5.1.0 (updated March 2011). The Cochrane Collaboration, 2011. Available from handbook.cochrane.org.

\section{Divine 1992}

Divine GW, Brown JT, Frazier LM. The unit of analysis error in studies about physicians' patient care behavior. Journal of General Internal Medicine 1992;7(6):623-9.

\section{Donner 2002}

Donner A, Klar N. Issues in the meta-analysis of cluster randomized trials. Statistics in Medicine 2002;21(19):2971-80.

\section{Egger 1997}

Egger M, Davey Smith G, Schneider M, Minder C. Bias in meta-analysis detected by a simple, graphical test. $B M J$ 1997;315(7109):629-34.

\section{Elbourne 2002}

Elbourne D, Altman DG, Higgins JPT, Curtina F, Worthington HV, Vaile A. Meta-analyses involving cross-over trials: methodological issues. International Journal of Epidemiology 2002;31(1):140-9.

\section{EuroQol Group 1990}

EuroQol Group. EuroQol - a new facility for the measurement of health-related quality of life. Health Policy 1990;16(3):199-208.

\section{Fioravanti 2005}

Fioravanti M, Carlone O, Vitale B, Cinti ME, Clare L. A metaanalysis of cognitive deficits in adults with a diagnosis of schizophrenia. Neuropsychology Review 2005;15(2):73-95.

\section{Furukawa 2006}

Furukawa TA, Barbui C, Cipriani A, Brambilla P, Watanabe N. Imputing missing standard deviations in meta-analyses can provide accurate results. Journal of Clinical Epidemiology 2006;59(1):7-10.

\section{Guhne 2015}

Gühne U, Weinmann S, Arnold K, Becker T, Riedel-Heller SG. S3 guideline on psychosocial therapies in severe mental illness: evidence and recommendations. European Archives of Psychiatry and Clinical Neuroscience 2015;265(3):173-88. [DOI: 10.1007/s00406-014-0558-9; PUBMED: 25384674]

\section{Gulliford 1999}

Gulliford MC, Ukoumunne OC, Chinn S. Components of variance and intraclass correlations for the design of communitybased surveys and intervention studies: data from the Health Survey for England 1994. American Journal of Epidemiology 1999;149(9):876-83.

\section{Higgins 2003}

Higgins JP, Thompson SG, Deeks JJ, Altman DG. Measuring inconsistency in meta-analyses. BMJ 2003;327(7414):557-60.

\section{Higgins 2011a}

Higgins JPT, Green S (editors). Chapter 7: Selecting studies and collecting data. In: Higgins JPT, Green S (editors). Cochrane Handbook for Systematic Reviews of Interventions Version
5.1.0 (updated March 2011). The Cochrane Collaboration, 2011. Available from handbook.cochrane.org.

\section{Higgins 2011b}

Higgins JPT, Altman DG, Sterne JAC (editors). Chapter 8: Assessing risk of bias in included studies. In: Higgins JPT, Green $\mathrm{S}$ (editors). Cochrane Handbook for Systematic Reviews of Interventions Version 5.1.0 (updated March 2011). The Cochrane Collaboration, 2011. Available from handbook.cochrane.org.

\section{Hutton 2009}

Hutton JL. Number needed to treat and number needed to harm are not the best way to report and assess the results of randomised clinical trials. British Journal of Haematology 2009;146(1):27-30

\section{Kay 1986}

Kay SR, Opler LA, Fiszbein A. Positive and Negative Syndrome Scale (PANSS) Manual. North Tonawanda, NY: Multi-Health Systems, 1986.

\section{Kielhofner 2008}

Kielhofner G. A Model of Human Occupation: Theory and Application. 4th Edition. Baltimore: Williams and Wilkins, 2008.

\section{Law 2005}

Law M, Baptiste S, Carswell A, McColl MA, Polatajko HJ, Pollock N. Canadian Occupational Performance Measure. 4th Edition. Ottawa: CAOT Publications ACE, 2005.

\section{Leon 2006}

Leon AC, Mallinckrodt CH, Chuang-Stein C, Archibald DG, Archer GE, Chartier K. Attrition in randomized controlled clinical trials: methodological issues in psychopharmacology. Biological Psychiatry 2006;59(11):1001-5. [PUBMED: 16905632]

\section{Leucht 2005a}

Leucht S, Kane JM, Kissling W, Hamann J, Etschel E, Engel R. Clinical implications of Brief Psychiatric Rating Scale scores. British Journal of Psychiatry 2005;187:366-71. [PUBMED: 16199797]

\section{Leucht 2005b}

Leucht S, Kane JM, Kissling W, Hamann J, Etschel E, Engel RR. What does the PANSS mean?. Schizophrenia Research 2005;79(2-3):231-8. [PUBMED: 15982856]

\section{Loudon 2015}

Loudon K, Treweek S, Sullivan F, Donnan P, Thorpe KE, Zwarenstein M. The PRECIS-2 tool: designing trials that are fit for purpose. BMJ 2015;350:h2147. [DOI: 10.1136/bmj.h2147]

\section{Mairs 2004}

Mairs $\mathrm{H}$, Bradshaw T. Life skills training in schizophrenia. Biritish Journal of Occupational Therapy 2004;67(5):217-24.

\section{Mangalore 2007}

Mangalore R, Knapp R. Cost of schizophrenia in England. Journal of Mental Health Policy and Economics 2007;10(1):23-41. 


\section{Marshall 2000}

Marshall M, Lockwood A, Bradley C, Adams C, Joy C, Fenton M. Unpublished rating scales: a major source of bias in randomised controlled trials of treatments for schizophrenia. British Journal of Psychiatry 2000;176:249-52.

\section{Marwaha 2007}

Marwaha S, Johnson S, Bebbington P, Stafford M, Angermeyer MC, Brugha T, et al. Rates and correlates of employment in people with schizophrenia in the UK, France and Germany. British Journal of Psychiatry 2007;191:30-7.

\section{McGrath 2008}

McGrath J, Saha S, Chant D, Welham J. Schizophrenia: a concise overview of incidence, prevalence, and mortality. Epidemiologic Reviews 2008;30:67-76.

\section{McGrath 2009}

McGrath JJ, Susser ES. New directions in the epidemiology of schizophrenia. Medical Journal of Australia 2009;190(Suppl 4):S7-9.

\section{Murray 2013}

Murray CJ, Richards MA, Newton JN, Fenton KA, Anderson HR, Atkinson C, et al. UK health performance: findings of the Global Burden of Disease Study 2010. Lancet 2013;381(9871):997-1020.

\section{Nagle 2002}

Nagle S, Cook J, Polatajko H. I'm doing as much as I can: occupational choices of persons with mental illness. Journal of Occupational Science 2002;9(2):72-81.

\section{NICE 2014}

National Institute for Health and Care Excellence (NICE). Psychosis and schizophrenia in adults: full guideline. guidance.nice.org.uk/CG178/Guidance/pdf/English (accessed 12 October 2015).

\section{NIMH 2014}

National Institute of Mental Health. Schizophrenia. www.nimh.nih.gov/health/topics/schizophrenia/index.shtml (accessed 1 October 2015).

\section{Overall 1962}

Overall JE, Gorham DR. The brief psychiatric rating scale. Psychological Reports 1962;10:799-812.

\section{Overall 1988}

Overall JE, Gorham DR. The Brief Psychiatric Rating Scale (BPRS): recent developments in ascertainment and scaling. Psychopharmacology Bulletin 1988;24:97-9.

\section{Parkinson 2006}

Parkinson S, Forsyth K, Kielhofner G. The Model of Human Occupation Screening Tool (Version 2.0). Chicago: Model of Human Occupation Clearinghouse, University of Illinois at Chicago, 2006

\section{Saraswat 2006}

Saraswat N, Rao K, Subbakrishna DK, Gangadhar BN. The Social Occupational Functioning Scale (SOFS): a brief measure of functional status in persons with schizophrenia. Schizophrenia Research 2006;81(2-3):301-9.

\section{Schünemann 2011}

Schünemann HJ, Oxman AD, Vist GE, Higgins JPT, Deeks JJ, Glasziou P, et al. Chapter 12: Interpreting results and drawing conclusions. In Higgins JPT, Green S (editors). Cochrane Handbook for Systematic Reviews of Interventions Version 5.1.0 (updated March 2011). The Cochrane Collaboration, 2011. Available from handbook.cochrane.org.

\section{Shokraneh 2017}

Shokraneh F, Adams CE. Study-based registers of randomized controlled trials: starting a systematic review with data extraction or meta-analysis. Biolmpacts 2017;7(4):209-17. [DOI 10.15171/bi.2017.25]

\section{Shokraneh 2018}

Shokraneh F, Adams CE. Gallstone, snake venom and witchcraft for schizophrenia: the challenges of classifying [schizophrenia] trials. Evidence-Based Medicine 2018;23(Suppl 1):A18. [DOI: 10.1136/bmjebm-2018-111024.36]

\section{Smith 2014}

Smith R, De Witt P, Franzen D, Pillay M, Wolfe N, Davies C. Occupational performance factors perceived to influence the readmission of mental health care users diagnosed with schizophrenia. South Africa Journal of Occupational Therapy 2014;44(1):51-6.

\section{Sterne 2011}

Sterne JAC, Egger M, Moher D (editors). Chapter 10: Addressing reporting biases. In: Higgins JPT, Green S (editors). Cochrane Handbook for Systematic Reviews of Interventions Version 5.1.0 (updated March 2011). The Cochrane Collaboration, 2011. Available from handbook.cochrane.org.

\section{Tungpunkom 2012}

Tungpunkom P, Maayan N, Soares-Weiser K. Life skills programmes for chronic mental illnesses. Cochrane Database of Systematic Reviews 2012, Issue 1. [DOI: 10.1002/14651858.CD000381.pub3]

\section{Ukoumunne 1999}

Ukoumunne OC, Gulliford MC, Chinn S, Sterne JAC, Burney PGJ. Methods for evaluating area-wide and organisation-based intervention in health and health care: a systematic review. Health Technology Assessment 1999;3(5):1-75.

\section{Urlic 2010}

Urlic K, Lentin P. Exploration of the occupations of people with schizophrenia. Australian Occupational Therapy Journal 2010;57(5):310-7.

\section{WFOT 2010}

World Federation of Occupational Therapists Council. Statement on occupational therapy. www.wfot.org/Portals/0/ PDF/STATEMENT\%200N\%200CCUPATIONAL\%20THERAPY \%20300811.pdf (accessed 12 August 2016). 


\section{Whiteford 2013}

Whiteford HA, Degenhardt L, Rehm J, Baxter AJ, Ferrari AJ, Erskine $\mathrm{HE}$, et al. Global burden of disease attributable to mental and substance use disorders: findings from the Global Burden of Disease Study 2010. Lancet 2013;382(9904):1575-86.

\section{WHO 2015}

World Health Organization. Mental health: schizophrenia. www.who.int/mental_health/management/schizophrenia/en/ (accessed 12 August 2016).

\section{CHARACTERISTICS OF STUDIES}

\section{Characteristics of excluded studies [ordered by study ID]}

\section{Xia 2009}

Xia J, Adams CE, Bhagat N, Bhagat V, Bhoopathi P, El-Sayeh H, et al. Loss to outcomes stakeholder survey: the LOSS study. Psychiatric Bulletin 2009;33(7):254-7.

* Indicates the major publication for the study

\begin{tabular}{|c|c|}
\hline Study & Reason for exclusion \\
\hline \multirow[t]{4}{*}{ Anqiong 2010} & Allocation: unclear \\
\hline & Participants: people with senile schizophrenia \\
\hline & Intervention: recreational therapy \\
\hline & $\begin{array}{l}\text { Reason for exclusion: does not compare interventions delivered by occupational therapists vs } \\
\text { those delivered by others }\end{array}$ \\
\hline \multirow[t]{4}{*}{ Campbell 1983} & Allocation: randomised \\
\hline & Participants: people with schizophrenia \\
\hline & Intervention: occupational therapy vs no occupational therapy \\
\hline & $\begin{array}{l}\text { Reason for exclusion: does not compare interventions delivered by occupational therapists vs } \\
\text { those delivered by others }\end{array}$ \\
\hline \multirow[t]{4}{*}{ Chan 2007} & Allocation: randomised \\
\hline & Participants: people with schizophrenia \\
\hline & $\begin{array}{l}\text { Intervention: Transforming Relapse and Instilling Prosperity (TRIP) programme vs ward occupa- } \\
\text { tional therapy }\end{array}$ \\
\hline & $\begin{array}{l}\text { Reason for exclusion: does not compare interventions delivered by occupational therapists vs } \\
\text { those delivered by others }\end{array}$ \\
\hline \multirow[t]{4}{*}{ Debin 2015} & Allocation: randomised \\
\hline & Participants: people with schizophrenia \\
\hline & Intervention: occupational therapy vs phytotherapy \\
\hline & $\begin{array}{l}\text { Reason for exclusion: does not compare interventions delivered by occupational therapists vs } \\
\text { those delivered by others }\end{array}$ \\
\hline \multirow[t]{4}{*}{ Du 2001} & Allocation: randomised \\
\hline & Participants: people with schizophrenia \\
\hline & Intervention: sulpiride plus occupational therapy vs occupational therapy alone \\
\hline & $\begin{array}{l}\text { Reason for exclusion: does not compare interventions delivered by occupational therapists vs } \\
\text { those delivered by others }\end{array}$ \\
\hline
\end{tabular}




\begin{tabular}{ll}
\hline Study & Reason for exclusion \\
\hline Gao 2004 & Allocation: randomised \\
& Participants: people with schizophrenia \\
& Intervention: occupational therapy vs no occupational therapy \\
& Reason for exclusion: does not compare interventions delivered by occupational therapists vs \\
& those delivered by others \\
& Allocation: randomised \\
& Participants: people with schizophrenia \\
Intervention: Mode of Human Occupation (MOHO)-informed rehabilitation training vs routine treat- \\
ment \\
Reason for exclusion: does not compare interventions delivered by occupational therapists vs \\
those delivered by others
\end{tabular}

Participants: people with schizophrenia

Intervention: participant-chosen activities vs occupational therapist-chosen activities

Reason for exclusion: does not compare interventions delivered by occupational therapists vs those delivered by others

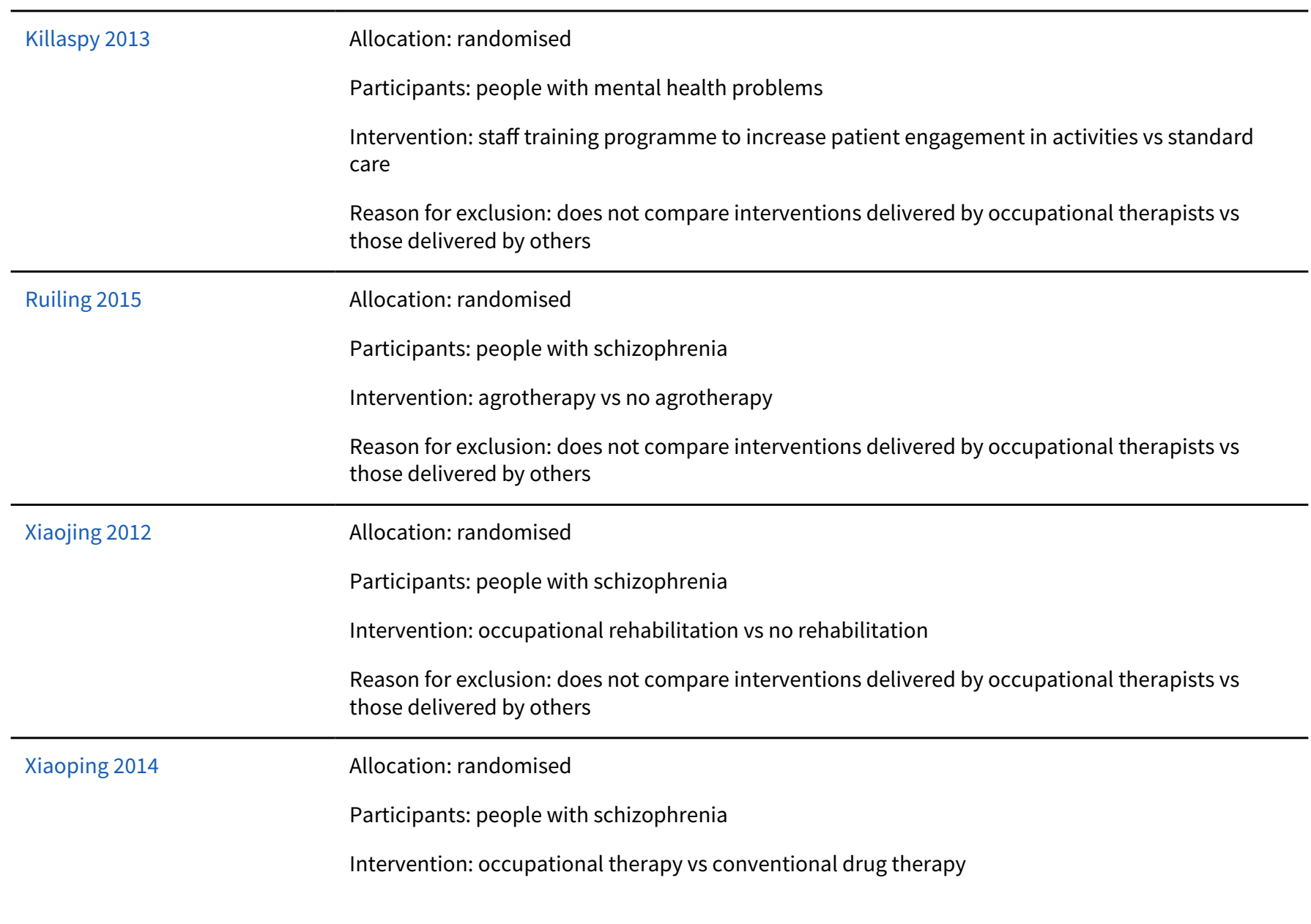




\begin{tabular}{ll}
\hline Study & Reason for exclusion \\
\hline Xiong 2013 & $\begin{array}{l}\text { Reason for exclusion: does not compare interventions delivered by occupational therapists vs } \\
\text { those delivered by others }\end{array}$ \\
\hline & Allocation: randomised \\
& Participants: people with schizophrenia \\
& Intervention: outdoor treatment vs manual treatment \\
& $\begin{array}{l}\text { Reason for exclusion: does not compare interventions delivered by occupational therapists vs } \\
\text { those delivered by others }\end{array}$ \\
\hline Aanyan 2016 & $\begin{array}{l}\text { Allocation: randomised } \\
\text { Participants: people with schizophrenia } \\
\text { Intervention: } 3 \text {-level rehabilitation work therapy vs general work-entertainment therapy } \\
\text { Reason for exclusion: does not compare interventions delivered by occupational therapists vs } \\
\text { those delivered by others }\end{array}$ \\
\hline
\end{tabular}

\section{ADDITIONAL TABLES}

Table 1. Reviews suggested by excluded studies

\section{Intervention \#1}

Intervention \#2

\section{Absolute effects}

\section{Occupational therapy}

$\begin{array}{ll}\text { Campbell 1983, Gao } & \text { Occupational therapy } \quad \text { No occupational therapy } \\ 2004 \text { Xiaoping 2014* } & \end{array}$

\section{Occupational therapy-mediated treatment}

\begin{tabular}{ll}
\hline Killaspy $2013 \quad \begin{array}{l}\text { Staff training programme to increase patient engagement in ac- } \\
\text { tivities }\end{array}$ & Standard care
\end{tabular}
tivities

\section{Other occupational-related comparisons}

\begin{tabular}{|c|c|c|}
\hline Anqiong $2010^{\star \star}$ & Recreational therapy & Usual care \\
\hline Ruiling 2015 & Agrotherapy & \\
\hline Guofu 2013 & $\begin{array}{l}\text { Mode of Human Occupation (MOHO)-informed rehabilitation } \\
\text { training }\end{array}$ & \\
\hline Xiaojing 2012 & Occupational rehabilitation & \\
\hline
\end{tabular}

\section{Comparative effects}

\section{Occupational therapy}


Table 1. Reviews suggested by excluded studies (Continued)
Chan 2007
Ward occupational therapy
Transforming Relapse and Instilling
Prosperity (TRIP) programme

Debin $2015 \quad$ Occupational therapy Phytotherapy

\section{Supplementation of occupational therapy}

\begin{tabular}{ll}
\hline Du 2001 & Sulpiride plus occupational therapy
\end{tabular}

\section{Occupational therapist-mediated treatments}

\begin{tabular}{lll}
\hline Hoshii 2013 & Occupational therapist-chosen activities & Participant-chosen activities \\
\hline Other occupational-related comparisons & \\
\hline Xiong 2013 & Outdoor treatment & Manual treatment \\
\hline Yanyan 2016 & 3-level rehabilitation work therapy & General work-entertainment therapy
\end{tabular}

*We think it likely that both groups received the usual care of antipsychotic medications.

${ }^{\star *}$ Schizophrenia of old age

\section{Table 2. Design of future trials}

\begin{tabular}{ll}
\hline Methods & $\begin{array}{l}\text { Allocation: randomised - clearly described generation of sequence and concealment of allocation } \\
\text { Blinding: not possible as both participants and therapists know who is delivering the intervention, } \\
\text { but outcome assessors should be independent and blinded to group allocation } \\
\text { Duration: } 3 \text { months minimum }\end{array}$ \\
\hline Setting & Hospital or community \\
\hline Participants & $\begin{array}{l}\text { Diagnosis: schizophrenia (operational criteria) } \\
\mathrm{N}=300^{*}\end{array}$ \\
& $\begin{array}{l}\text { Age: children ( } \leq 18 \text { years) or adults ( }>18 \text { years), or both using stratified randomisation to ensure } \\
\text { balanced distribution and sample size calculation adjusted accordingly } \\
\text { Gender: both } \\
\text { History: any }\end{array}$
\end{tabular}

Interventions

1. Occupational therapy delivered by occupational therapists. $\mathrm{N}=150$

2. Occupational therapy delivered by non-specialists. $\mathrm{N}=150$

The occupational therapy should be delivered either at the individual or group level but not both unless they are equally distributed between groups, taken into account in the analyses, and power calculations for study sample sizes adjusted accordingly.

To maintain relevance for practice, concomitant therapies such as pharmacotherapy should be permitted providing they are equally distributed between groups.

\section{Outcomes}

Activities of daily living - proportion with clinical important change ${ }^{\star \star}$

Clinical global state - relapse ${ }^{\star \star}$

Service utilisation outcomes - hospital admission**

Extrapyramidal adverse effects**

Economic outcomes - incremental cost-effectiveness ratio 
Table 2. Design of future trials (Continued)

Notes

Study designs should follow Standard Protocol Items: Recommendations for Interventional Trials (SPIRIT) guidelines for the development of trial protocols (Chan 2013); CONSORT guidelines for the design of trials of non-pharmacological interventions (Boutron 2008); and Pragmatic Explanatory Continuum Indicator Summary (PRECIS-2) guidelines for the design of trials that are fit for purpose (Loudon 2015), as appropriate.

*The number of participants needed to gain sufficient power to identify a $10 \%$ difference between groups for the primary outcome depends on the specific primary outcome selected and its prevalence/magnitude. $\mathrm{N}=300$ is the approximate size of study to detect a $10 \%$ difference in improvement with $80 \%$ certainty.

${ }^{\star \star}$ Primary outcome

\section{CONTRIBUTIONS OF AUTHORS}

All authors contributed to the Abstract, Background, Results, Discussion, and Authors' conclusions.

Sally Spencer and Karen Morris contributed to the Methods.

Karen Morris and Graeme Reid conducted the study selection.

\section{DECLARATIONS OF INTEREST}

Karen Morris: none known.

Graeme Reid: none known.

Sally Spencer is an editor for the Cochrane Airways Group and the Cochrane Developmental and Cognitive Improvement Group.

\section{SOURCES OF SUPPORT}

\section{Internal sources}

- Edge Hill University, UK.

Employs review author Sally Spencer

- University of Cumbria, UK.

Employs review author Karen Morris

- North West Boroughs Healthcare NHS Foundation Trust, UK.

Employs review author Graeme Reid

\section{External sources}

- No external source of support provided, Other.

\section{DIFFERENCES BETWEEN PROTOCOLANDREVIEW}

There are no differences between the methods in the published protocol and the completed review.

\section{INDEX TERMS}

\section{Medical Subject Headings (MeSH)}

${ }^{\star}$ Occupational Therapists; ${ }^{\star}$ Specialization; Occupational Therapy [ ${ }^{\star}$ methods]; Schizophrenia [*therapy]

\section{MeSH check words}

Humans 